\title{
Possible Similarity Cases for Internal Heat Generation, Thermal Radiation and Free Convection of Unsteady Boundary Layer Flow Over a Vertical Plate
}

\author{
N. M. Ridwan Zahed ${ }^{1}$, Md. Yeakub Ali ${ }^{1, ~}$, Md. Jashim Uddin ${ }^{1,2}$, Mohammed Nasir Uddin ${ }^{1}$ \\ ${ }^{1}$ Department of Mathematics, Chittagong University of Engineering \& Technology, Chittagong, Bangladesh \\ ${ }^{2}$ Department of Electrical \& Electronic Engineering, International Islamic University Chittagong, Chittagong, Bangladesh
}

Email address:

ali69cuet@gmail.com (Md. Y. Ali)

${ }^{*}$ Corresponding author

\section{To cite this article:}

N. M. Ridwan Zahed, Md. Yeakub Ali, Md. Jashim Uddin, Mohammed Nasir Uddin. Possible Similarity Cases for Internal Heat Generation, Thermal Radiation and Free Convection of Unsteady Boundary Layer Flow Over a Vertical Plate. Applied and Computational Mathematics. Vol. 6, No. 1, 2017, pp. 60-67. doi: 10.11648/j.acm.20170601.15

Received: January 21, 2017; Accepted: February 6, 2017; Published: March 4, 2017

\begin{abstract}
In this paper we analyze the effects of free convection for internal heat generation and thermal radiation of an unsteady boundary layer flow over a vertical plate. We have applied free parameter method to obtain possible similarity cases. One of these cases have been investigated analytically and numerically. In this case, the governing non-linear partial differential equations are transformed into a system of coupled non-linear ordinary differential equations using similarity transformations and then solved numerically using the Runge-Kutta method with shooting technique for better accuracy. The effects of the governing parameters on the flow and thermal fields are investigated numerically and displayed graphically. The skin-friction coefficient, heat transfer coefficient have also been obtained and presented in tabular form.
\end{abstract}

Keywords: Boundary Layer, Heat Generation, Thermal Radiation, Buoyancy Force, Similarity Solution, Vertical Plate

\section{Introduction}

The fluid flow caused only due to the density differences resulting from temperature gradients without the assistance of any external force is termed as natural or free convection flow. Free convective flow involving heat transfer occurs frequently which makes a difference between the temperature of land and air and arises to a combined flow patterns. This may seen in engineering and manufacturing sectors, Nuclear power plants, gas turbines and various propulsion devices for aircraft, missiles, satellites and space vehicles. Thermal radiation, which is a natural phenomena in the nature that has an important significance. The protection of nature and ozone layer surrounding the earth are such example. Combination of heat and mass transfer plays an important role in designing of chemical processing equipment, formation and dispersion of fog, distribution of temperature and moisture over agricultural fields close to a surface. Illingworth [1] studied the problem of unsteady laminar flow of a gas near an infinite flat plate. He obtained solutions which are available only with Prandtl number unity and under transient conditions of step change in the surface temperature. Hellums and Churchill [2], Callahan and Marner [3] studied the problem with mass transfer using the finite difference method. Menold and Yang [4] presented general solution to the same problem for certain class of surface temperature variations. Williams et al. [5] studied the unsteady free convection flow over a vertical flat plate under the assumption of variations of the wall temperature. They found possible semi-similar solutions for wall temperature distributions. Boutros et al [6] solved the steady freeconvective boundary layer flow on a non-isothermal vertical flat plate. Many studies were made on the steady free convective boundary-layer flow on moving vertical plates considering the effects of buoyancy forces on the boundary layer. Kumari et al. [7] investigated the unsteady free convection flow over a continuous moving vertical plate. Bataller [8] showed a numerical solution for the combined effects of thermal radiation and convective surface heat transfer on the laminar boundary layer about a flat-plate in a 
uniform stream of fluid and about a moving plate in a quiescent ambient fluid. Hong et al. [9], Chen and Lin [10] and Jaisawal and Soundalgekar [11] studied the free convection in a porous medium with high porosity. After a pioneering work of Sakiadis $[12,13]$ the study of flow and heat transfer characteristics past continuous stretching surfaces has drawn considerable attention. The effects of slip boundary condition on the flow of Newtonian fluid due to a stretching sheet were explained by Andersson [14] and Wang [15]. Tsou et al [16] showed that the Sakiadis flow is physically realizable under laboratory condition and they determined the heat transfer rates for certain values of the Prandtl number. A vast literature of similarity solution has appeared in the fluid mechanics of heat transfer and mass transfer. Ali et al [17] investigated the similarity solution of unsteady free convective laminar boundary flow around a vertical heated curvilinear surface. The combined effect of increasing the Prandtl number and the Grashof number reduces the thermal boundary layer thickness along the plates which is vita in engineering application. Aziz [18] studied similarity solution for laminar thermal boundary layer over a flat plate with a convective surface boundary condition. More over Magyari [19] studied the similar problem which was extended by Ishak [20]. Makinde and Olanrewaju [21] investigated the buoyancy effects on thermal boundary layer over a vertical plate with a convective surface boundary condition. An extensive literature that deals with flows in the presence of radiation Raptis et al [22] studied the effect of thermal radiation on the MHD flow of viscous fluid past a semi-infinite stationary plate. Hayat et al [23] extended the wok of Raptis et al [22]. The present study is the unsteady work of Olanrewaju et al [24] to include the heat generation and the thermal radiation which has application in industry, science and engineering. In the present study we discuss possible similarity cases for unsteady boundary layer flow over a vertical plate. We provide analytical and numerical solution of possible similarity cases for the unsteady freeconvection flow over a vertical plate. In the similarity case the governing partial differential equations are reduced to a set of ordinary differential equations with the appropriate boundary conditions. The obtained differential equations are solved numerically using the shooting method.

\section{Mathematical Formulation}

Two-dimensional unsteady incompressible fluid flow over a vertically heated plate is considered with a stream of cold fluid at temperature $T_{\infty}$ moves over the right surface of the plate with a uniform velocity $U_{\infty}$. The left surface of the plate is heated by convection from a hot fluid at temperature $T_{f}$ which provides a heat transfer coefficient $h_{f}$. Assuming the Boussinesq approximation the governing equations for the problem are

$$
\begin{gathered}
\frac{\partial u}{\partial x}+\frac{\partial v}{\partial y}=0 \\
\frac{\partial u}{\partial t}+u \frac{\partial u}{\partial x}+v \frac{\partial u}{\partial y}=v \frac{\partial^{2} u}{\partial y^{2}}+g \beta\left(T-T_{\infty}\right) \\
\frac{\partial T}{\partial t}+u \frac{\partial T}{\partial x}+v \frac{\partial T}{\partial y}=\alpha \frac{\partial^{2} T}{\partial y^{2}}+Q_{1}\left(T-T_{\infty}\right)-\frac{\alpha}{\kappa} \frac{\partial q_{r}}{\partial y}
\end{gathered}
$$

Where $\mathrm{u}$ and $\mathrm{v}$ are the $\mathrm{x}$ (along the plate) and the $\mathrm{y}$ (normal to the plate) components of the velocities, respectively. $\mathrm{T}$ is the temperature, $v$ is the kinematics viscosity of the fluid, and $\alpha$ is the thermal diffusivity of the fluid and $\beta$ is thermal expansion coefficient. $Q_{1}$ is the heat release per unit mass $g$ is the gravitational acceleration and $q_{r}$ is the radiative heat flux respectively. The velocity boundary conditions can be expressed as

$$
\begin{aligned}
& y \rightarrow 0, u=0, v=0, T=T_{f} \\
& y \rightarrow \infty, u=U_{\infty}, T=T_{\infty}
\end{aligned}
$$

The radiative heat flux $q_{r}$ is described by Roseland approximation such that

$$
q_{r}=-\frac{4 \sigma}{3 \kappa} \frac{\partial T^{4}}{\partial y}
$$

Where $\sigma$ and $\mathrm{K}$ are the Stefan- Boltzmann constant and the mean absorption coefficient.

Following Chamkha [25], we assume that the temperature differences within the flow are sufficiently small so that the $T^{4}$ can be expressed as a linear function after using Taylor series to expand $T^{4}$ about the free stream temperature $T_{\infty}$ and neglecting higher-order terms. This result is the following approximation

$$
T^{4} \approx 4 T_{\infty}^{3} T-3 T_{\infty}^{4}
$$

Using (5) and (6)

$$
\frac{\partial q_{r}}{\partial y}=-\frac{4 \sigma}{3 K} T_{\infty}^{3} \theta^{/ /}\left(T_{f}-T_{\infty}\right) \frac{U_{\infty}}{v \gamma^{2}}
$$

To reduce the above system of equations into suitable form, we used the free parameter method. Hence the following substitutions are introduced

$$
x=\xi, t=\tau, \eta=y \sqrt{\frac{U_{\infty}}{v}} \cdot \frac{1}{\gamma(x, t)}, f^{\prime}(\eta)=\frac{u}{U_{\infty}}, \psi=\sqrt{U_{\infty} v} f(\eta) \gamma(x, t),
$$

where prime denotes differentiation with respect to $\eta$ Equation(1-4) reduces to 


$$
\begin{gathered}
f^{\prime \prime \prime}+a_{0} f^{\prime \prime} f+a_{1} \eta f^{\prime \prime}+G r \theta=0 \\
\left(\operatorname{Pr}^{-1}+R a\right) \theta^{\prime \prime}+\left(a_{1} \eta+a_{0} f\right) \theta^{\prime}+Q \theta=0
\end{gathered}
$$

with the boundary condition

$$
\begin{array}{ll}
f(0)=f^{\prime}(0)=0, \theta(0)=1 & \text { at } \eta=0 \\
f^{\prime}(\infty)=1, \theta(\infty)=0 & \text { at } \eta \rightarrow \infty
\end{array}
$$

Where,

$$
\begin{gathered}
\gamma \frac{\partial \gamma}{\partial \xi}=a_{0}, \frac{\gamma}{U_{\infty}} \frac{\partial \gamma}{\partial \tau}=a_{1} \\
\text { Modified Radiation number } \quad R a=\frac{16}{3} \frac{\sigma \alpha}{\kappa K \nu} T_{\infty}{ }^{3}
\end{gathered}
$$

Modified heat release parameter, $Q=Q_{1} \frac{\gamma^{2}}{U_{\infty}}$

$$
\text { Prandtl number } \quad, P a=\frac{v}{\alpha}
$$

$$
\text { Modified Grashof number, } G r=\frac{\gamma^{2} g \beta}{U_{\infty}^{2}}\left(T_{f}-T_{\infty}\right)
$$

For the momentum and energy equations to have a similarity solution, the parameter $G r, \operatorname{Pr}, Q, R a, a_{0}, a_{1}$ are defined.

Similar solution for equation (9-10) exists only when all constants are finite and independent of $\xi$ and $\tau$. We obtain the following relationship.

$$
\begin{gathered}
\frac{\gamma^{2}}{2}=a_{0} \xi+A(\tau) \\
\frac{d A}{d \tau}=a_{1} U_{\infty} \\
\frac{\gamma^{2}}{2 U_{\infty}}=a_{1} \tau+B(\xi) \\
\frac{d B}{d \xi}=\frac{a_{0}}{U_{\infty}}
\end{gathered}
$$

Taking the product of $\frac{d A}{d \tau}$ and $\frac{d B}{d \xi}$, we obtain

$$
\frac{d A}{d \tau} \cdot \frac{d B}{d \xi}=a_{0} a_{1}
$$

The form of similarity solutions wholly depend on equation (21), which leads to different possible similarity cases. In this paper we discuss three possible cases analytically and also we discussed the case, $\frac{d A}{d \tau}=0, \frac{d B}{d \xi} \neq 0$ numerically.

When $\frac{d A}{d \tau}=0, \frac{d B}{d \xi}=0$ :

From (12) we have

$$
\gamma \gamma_{\xi}=a_{0}
$$

From (17) and (19),

$$
U_{\infty}=\frac{a_{0} \xi+A(\tau)}{a_{1} \tau+B(\xi)}
$$

From (12), we get

$$
\gamma \gamma_{\tau}=a_{1} U_{\infty}
$$

Use the equation (18), the similarity case, $\frac{d A}{d \tau}=0, \frac{d B}{d \xi}=0$ and we get $a_{0}=0, a_{1}=0$

Then the general equations reduces to

$$
\begin{gathered}
f^{\prime \prime}+G r \theta=0 \\
\left(\operatorname{Pr}^{-1}+R a\right) \theta^{\prime \prime}+Q \theta=0
\end{gathered}
$$

With the boundary conditions

$$
\begin{aligned}
& f(0)=f^{\prime}(0)=0, \theta(0)=1 \quad \text { at } \quad \eta \rightarrow 0 \\
& f^{\prime}(\infty)=1, \theta(\infty)=0 \quad \text { at } \quad \eta \rightarrow \infty
\end{aligned}
$$

When $\frac{d A}{d \tau} \neq 0, \frac{d B}{d \xi}=0$ :

From (17) and (19), we get $a_{0} \xi+A(\tau)=U_{\infty}\left(a_{1} \tau+B(\xi)\right)$

To maintain consistency both sides, we put $a_{0}=0$, then

$$
A(\tau)=U_{\infty}\left(a_{1} \tau+B(\xi)\right)
$$

And

$$
\frac{\gamma^{2}}{2 U_{\infty}}=a_{1} \tau+B(\xi)
$$

Where $B(\xi)=\mathrm{k}$ (constant)

$$
a_{1}=\frac{A(\tau)-k}{U_{\infty} \tau}
$$

Now, the general equation (9-10) reduces to

$$
\begin{gathered}
f^{\prime \prime \prime \prime}+a_{1} \eta f^{\prime \prime}+G r \quad \theta=0 \\
\left(\operatorname{Pr}^{-1}+R a\right) \theta^{\prime \prime}+a_{1} \eta \theta^{\prime}+Q \theta=0
\end{gathered}
$$

With the boundary condition 


$$
\begin{aligned}
& f(0)=f^{\prime}(0)=0, \theta(0)=1 \quad \text { at } \quad \eta \rightarrow 0 \\
& f^{\prime}(\infty)=1, \theta(\infty)=0 \quad \text { at } \quad \eta \rightarrow \infty
\end{aligned}
$$

When $\frac{d A}{d \tau}=0, \frac{d B}{d \xi} \neq 0$

From (17) and (19)

$$
a_{0} \xi+A(\tau)=U_{\infty}\left(a_{1} \tau+B(\xi)\right)
$$

$$
A(\tau)=k_{1} \text { (Constant) }
$$

To maintain consistency both sides, we put $a_{1}=0$, then

$$
a_{0}=\frac{U_{\infty} B(\xi)-k_{1}}{\xi}
$$

Where, $\frac{\gamma^{2}}{2}=a_{0} \xi+A(\tau)$

and $\frac{\gamma^{2}}{2}=a_{0} \xi+k_{1}$ (constant)

Then the general equations $(9-10)$ reduces to

$$
\begin{gathered}
f^{\prime \prime}+a_{0} f^{\prime \prime} f+G r \theta=0 \\
\left(\operatorname{Pr}^{-1}+R a\right) \theta^{\prime \prime}+a_{0} f \theta^{\prime}+Q \theta=0
\end{gathered}
$$

With the boundary condition

$$
\begin{aligned}
& f(0)=f^{\prime}(0)=0, \theta(0)=1 \quad \text { at } \eta \rightarrow 0 \\
& f^{\prime}(\infty)=1, \theta(\infty)=0 \quad \text { at } \eta \rightarrow \infty
\end{aligned}
$$

\section{Numerical Solution}

Systems of ordinary differential equations (10) to (11) with boundary conditions (12) and (13) are transformed into initial value problem and solved numerically by applying the Shooting method namely Nachtsheim-Swigert (1965) iteration technique (guessing the missing value) along with Runge-Kutta integration scheme. In the process of iteration the velocity, the temperature, the Skin friction coefficient $f(0)$ and Nusselt number $\theta^{\prime}(0)$ are also evaluated. The numerical results have been obtained and displayed in figures and tables for the selected parameters.

\section{Results and Discussion}

To obtain the solution of the differential equations $(32,33)$ with the boundary conditions, a numerical procedure based on Runge -Kutta sixth order integration scheme is implemented. The effects of various parameters on the flow and temperature profiles have been shown for the different values of modified Grashof number Gr, Prandtl number Pr, modified thermal Radiation $\mathrm{Ra}$, the unsteadiness parameter $a_{0}$ and the modified heat release parameter $Q$.

\subsection{Velocity Profiles}

The effects of various parameters on the non- dimensional velocity profiles within the boundary layer are shown in Figures
1-5. Generally the fluid velocity is zero at the plate surface and increases gradually away from the plate towards the free stream value satisfying boundary condition. The velocity profiles for different values of he Modified Grashof number Gr are described in Figure 1. It is observed that an increase in the Grashof number Gr leads to a rise in the values of velocity. In addition, the curves show that the peak value of velocity increases rapidly near the wall of the plate as the modified Grashof number $\mathrm{Gr}$ increases and it is understood that the rate of heat transfer at the plate surface increases with the increase of modified $G r$. Figure 2. represents the velocity profiles for different values of modified heat release parameter $Q$. The result shows that the velocity profiles increases with increase the values of modified heat release parameters and it is clear that the boundary layer thickness increases with increase the values of modified heat release parameter. The effects of the Prandtl number on the velocity profiles is shown in Figure 3. It is found out that the velocity profile decreases with the increasing values of Prandtl number Pr. When Prandtl number Pr increases the dynamic viscosity of the fluid increase and then slow down the velocity of the fluid. Figure 4 illustrates the variation of dimensionless velocity profiles for several value of thermal radiation. From this figure we observe that the velocity profiles monotonically increasing with the increase of thermal radiation. It is interesting to note that skin-friction and the rate of heat transfer at the plate surface increases with the increase of modified $\mathrm{Gr}$ and modified Ra but decreases with the increase of Pr. The effects of unsteadiness parameter $a_{0}$ on the velocity profiles is depicted by Figures 5. It is clearly seen that the velocity profiles increases with the increase of the values of the unsteadiness parameter $a_{0}$.

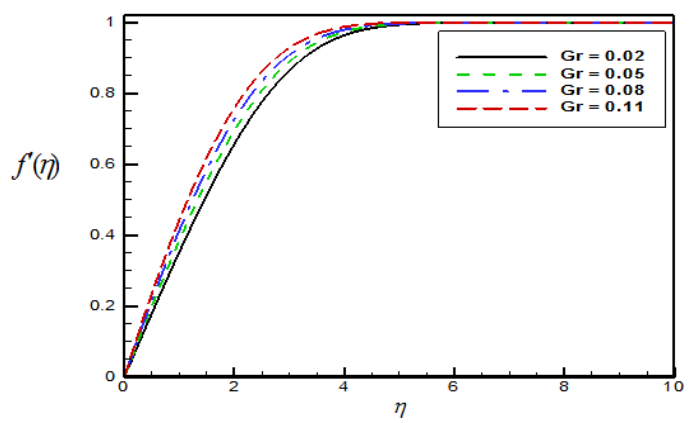

Figure 1. Velocity profiles for various values of modified $\mathrm{Gr}$ when $\mathrm{Pr}=$ $0.72, R a=0.01, a_{0}=0.50, Q=0.02$.

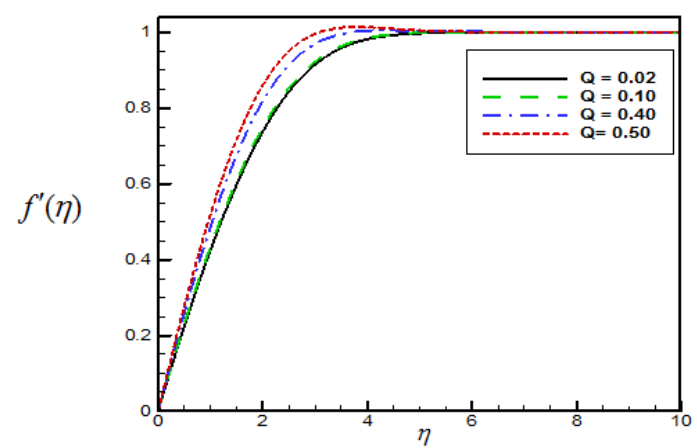

Figure 2. Velocity profiles for various values of modified $Q$, when $G r=0.02$, $\operatorname{Pr}=0.72, R a=0.01, a_{0}=0.50$. 


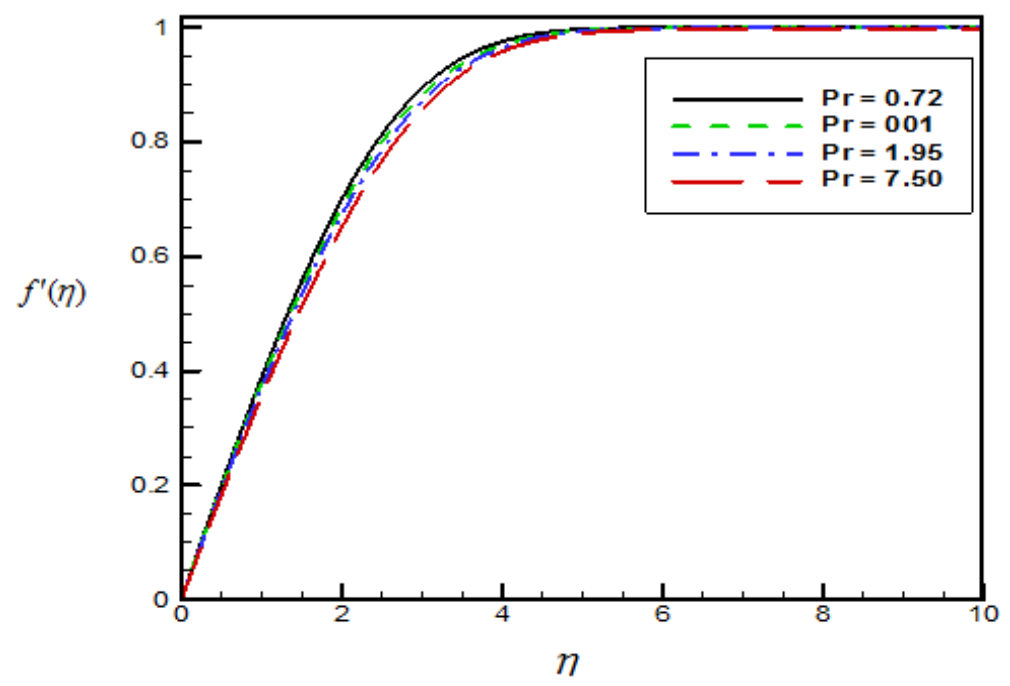

Figure 3. Velocity profiles for various values of $\operatorname{Pr}$ when $G r=0.02, R a=0.01, a_{0}=0.50, Q=0.02$.

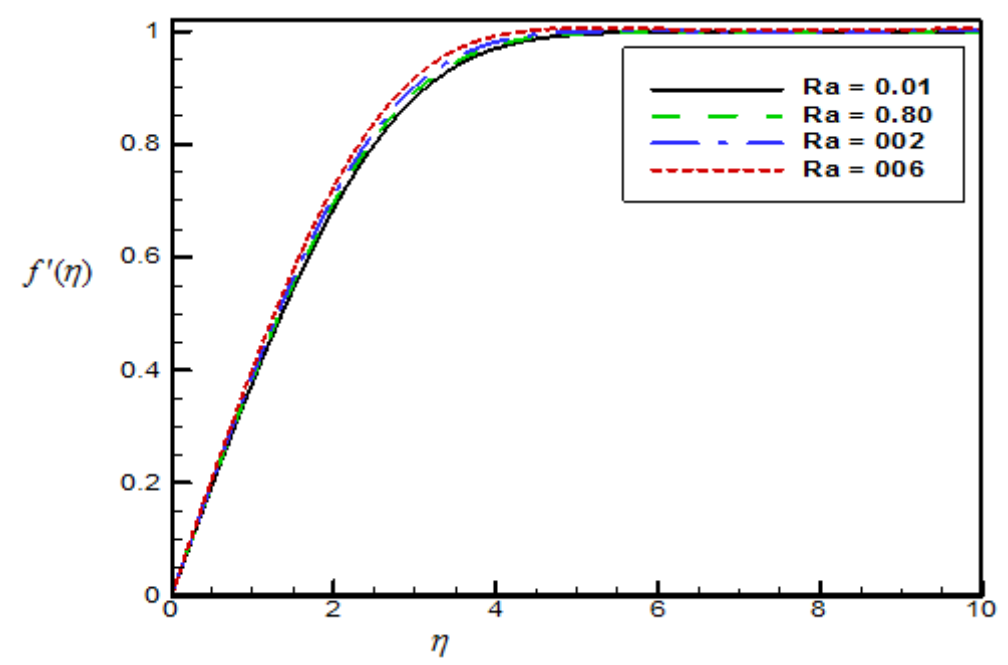

Figure 4. Velocity profiles for various values of modified Ra when $G r=0.02, \operatorname{Pr}=0.72, a_{0}=0.50, Q=0.02$.

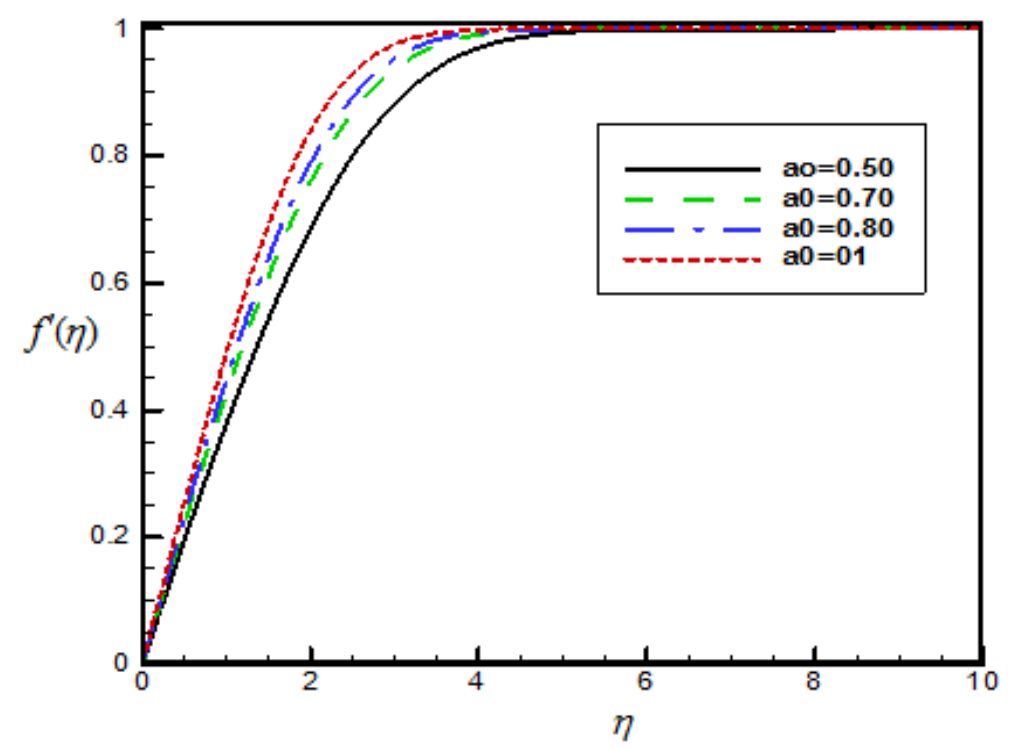

Figure 5. Velocity profiles for various values of $a_{0}$ when $G r=0.02, \operatorname{Pr}=0.72, R a=0.01, Q=0.02$. 


\subsection{Temperature Profiles}

Generally the fluid temperature is maximum at the plate surface and exponentially decreases to zero far away from the plate satisfying boundary condition. Figures 6-10 illustrate the behavior of the temperature profiles within the boundary layer. The influence of the Grashof number on the temperature profile is presented in Figure 6. It is observed that the variation of modified Grashof number (Gr) for temperature profile is decreasing. Figure 7 exhibits the behavior of dimensionless temperature profiles for different values of modified heat release $Q$. we see that the temperature profile increases with the increase of some values of modified heat release Q. Figure 8 represents the temperature profiles for different values of Prandtl number. A comparison of the curves from figure shows that a growing Prandtl number decreases the temperature of the flow field at all points. The temperature profiles for different values of the modified thermal radiation $R a$ are presented in Figure 9. We found out that the temperature profile increases with the increasing values of the thermal radiation which shows the thermal boundary layer thickness increases with the increases of modified thermal radiation $R a$. Figures 10 represents the variation of unsteadies parameters $a_{0}$. It is clearly seen that the temperature profiles decreases when there is an increase in the values of the unsteadiness parameter $a_{0}$, Hence internal heat generation parameter and radiation parameter enhances the thermal diffusion at an increase of Prandtl umber and the intensity of the buoyancy force slows down the rate of thermal diffusion with in the boundary layer.

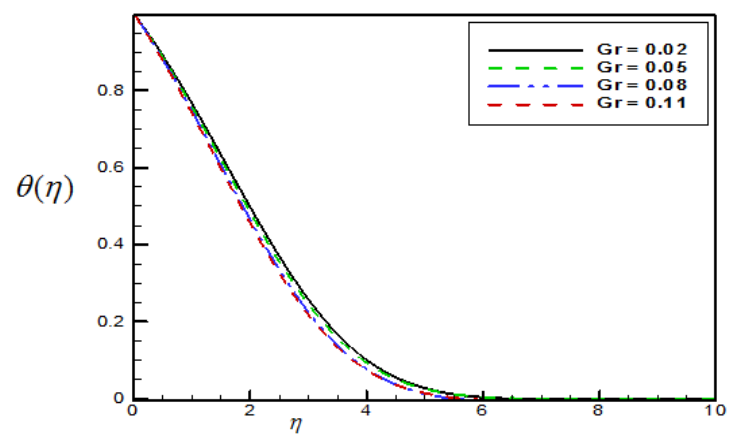

Figure 6. Temperature profiles for various values of modified $\mathrm{Gr}$ when $\mathrm{Pr}$ $=0.72, R a=0.01, a_{0}=0.50, a_{2}=0.02$.

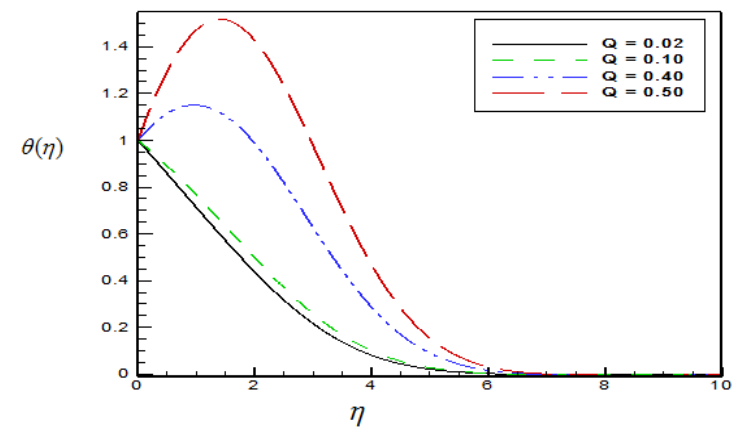

Figure 7. Temperature profiles for various values of modified $Q$ when $G r$ $=0.02, R a=0.01, a_{0}=0.50, \operatorname{Pr}=0.72$.

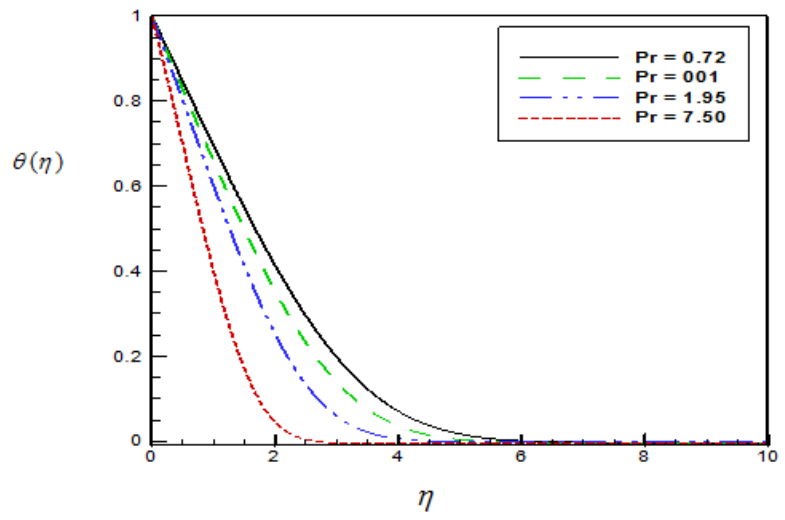

Figure 8. Temperature profiles for various values of $\mathrm{Pr}$ when $\mathrm{Gr}=0.02$, $R a=0.01, Q=0.02, a_{0}=0.50$.

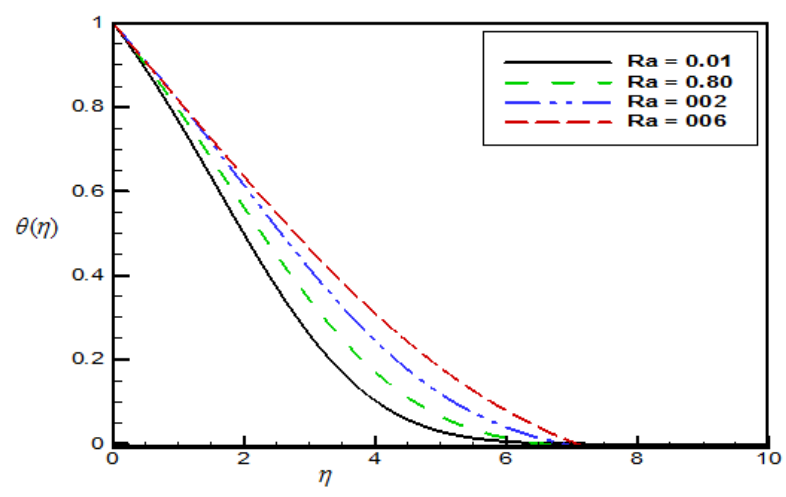

Figure 9. Temperature profiles for various values of modified $R a$ when $\mathrm{Gr}=$ $0.02, \operatorname{Pr}=0.72, a_{0}=0.50, Q=0.02$.

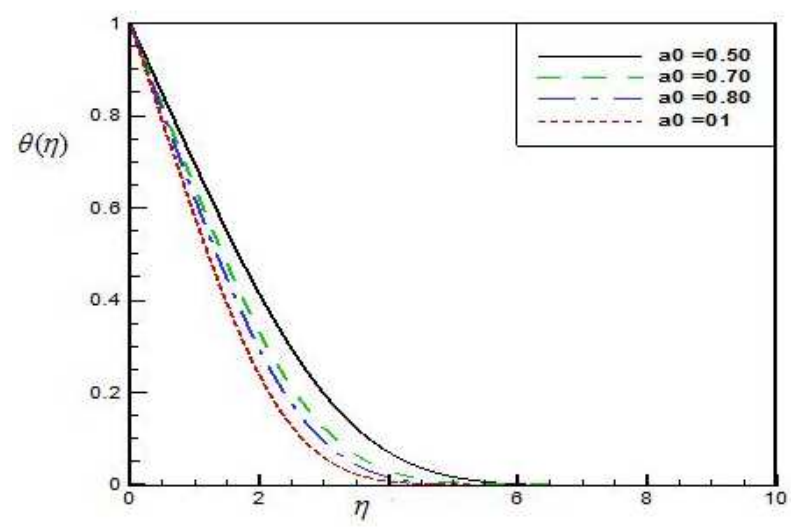

Figure 10. Temperature profiles for various values of $a_{0}$ when $\mathrm{Gr}=0.02, \mathrm{Pr}$ $=0.72, R a=0.01, Q=0.02$.

The numerical results are illustrated in Tables 1 to 5 for the effects of various parameters on the Skin-friction coefficient and local Nusselt number. The Skin-friction coefficient and the local Nusselt number represent in terms of $f^{\prime \prime}(0)$ and $-\theta^{\prime}(0)$ respectively. From the Tables 1,2 and 4 it is understood that the Skin-friction co-efficient increases with an increase of Modified $G r$, Modified $Q$ and Modified $R a$ while Nusselt number decreases for Modified $G r$ but increases for $Q$ and $R a$. However with the increase of prandtl number $\operatorname{Pr}$ decreases both the Skin-friction and Nusselt 
number are shown in table 3 . In Table 5, we see that the Skin-friction increases with the increase of $a_{0}$ but decreases the Nusselt number.

Table 1. Effects of Gr on Skin-friction $f^{\prime \prime}(0)$ and Nusselt number $-\theta^{\prime}(0)$ for the reference values in Figure. 1 .

\begin{tabular}{lll}
\hline $\mathbf{G r}$ & $\boldsymbol{f}^{\prime \prime}(0)$ & $-\boldsymbol{\theta}^{\prime}(0)$ \\
\hline 0.02 & 0.361427 & 0.299483 \\
0.05 & 0.403823 & 0.306352 \\
0.08 & 0.429233 & 0.311348 \\
0.11 & 0.471787 & 0.315286 \\
\hline
\end{tabular}

Table 2. Effects of $Q$ on Skin-friction $f^{\prime \prime}(0)$ and Nusselt number $-\theta^{\prime}(0)$ for the reference values in Figure.2.

\begin{tabular}{lll}
\hline $\mathbf{Q}$ & $\boldsymbol{f}^{\prime \prime}(0)$ & $-\boldsymbol{\theta}^{\prime}(0)$ \\
\hline 0.02 & 0.360808 & 0.282485 \\
0.10 & 0.363835 & -0.203956 \\
0.40 & 0.383098 & 0.190035 \\
0.50 & 0.399965 & 0.189635 \\
\hline
\end{tabular}

Table 3. Effects of Pr on Skin-friction $f^{\prime \prime}(0)$ and Nusselt number $-\theta^{\prime}(0)$ for the reference values in Figure. 3 .

\begin{tabular}{lll}
\hline $\operatorname{Pr}$ & $\boldsymbol{f}^{\prime \prime}(0)$ & $-\boldsymbol{\theta}^{\prime}(0)$ \\
\hline 0.72 & 0.361427 & 0.299483 \\
01 & 0.359120 & 0.335786 \\
1.95 & 0.352353 & 0.485293 \\
7.50 & 0.348475 & 0.636822 \\
\hline
\end{tabular}

Table 4. Effects of Ra on Skin-friction $f^{\prime \prime}(0)$ and Nusselt number $-\theta^{\prime}(0)$ for the reference values in Figure.4.

\begin{tabular}{lll}
\hline $\mathbf{R a}$ & $\boldsymbol{f}^{\prime \prime}(0)$ & $-\boldsymbol{\theta}^{\prime}(0)$ \\
\hline 0.01 & 0.361427 & 0.299483 \\
0.80 & 0.365233 & 0.249034 \\
02 & 0.373456 & 0.181487 \\
06 & 0.373610 & 0.176192 \\
\hline
\end{tabular}

Table 5. Effects of $a_{0}$ on Skin-friction $f^{\prime \prime}(0)$ and Nusselt number $-\theta^{\prime}(0)$ for the reference values in Figure.5.

\begin{tabular}{lll}
\hline $\boldsymbol{a}_{0}$ & $\boldsymbol{f}^{\prime \prime}(0)$ & $-\boldsymbol{\theta}^{\prime}(0)$ \\
\hline 0.50 & 0.362000 & 0.281635 \\
0.70 & 0.418202 & 0.337437 \\
0.80 & 0.443863 & 0.362230 \\
01 & 0.490006 & 0.410873 \\
\hline
\end{tabular}

\section{Conclusions}

Possible similarity cases has been presented for the unsteady free convection boundary layer flow over a vertical plate in viscous incompressible fluid. For the similarity case, $\frac{d A}{d \tau}=0, \frac{d B}{d \xi} \neq 0$, following conclusion are made:.

(a) With an increase of the Grashof number $G r$ the velocity profiles increases but the increase of $G r$ the temperature profiles decreases. It also seen that the values of skin-friction coefficient increases and but Nusselt number decreases.

(b) With an increase of Prandtl number $P r$ both the velocity and temperature profiles decreases. Also both the co-efficient of skin-friction and heat transfer decreases with the increase of the prandtl number.

(c) Increase of thermal radiation $Q$ both the velocity and the temperature profiles increases while both the Skinfriction co-efficient Nusselt number increases.

(d) The velocity and temperature profiles increases with the increase of heat generation parameters.

(e) Increase of unsteadies parameters $a_{0}$, the velocity profile increases while the temperature profiles decreases.

\section{References}

[1] C. R. Illingworth, Unsteady laminar flow of gas near an infinite flat plate, Proc. Camb. Philos. Soc. 46 (1950) 603613.

[2] J. D. Hellums, S. W. Churchill, Transient and steady state free and natural convection, numerical solutions, A. I. Ch. E. J. 8 (1962) 690-695.

[3] G. D. Callahan, W. J. Marner, Transient free convection with mass transfer on an isothermal vertical flat plate, Int. J. Heat Mass Transfer 19 (1976) 165-174.

[4] E. R. Menold, K. T. Yang, Asymptotic solutions for unsteady laminar free convection on a vertical plate, Trans. ASME. J. Appl. Mech. 29 (1962) 124-126.

[5] J. C. Williams, J. C. Mulligan, T. B. Rhyne, Semisimilar solution for unsteady free- convective boundary layer-flow on a vertical flat plate, J. Fluid Mech. 175 (1987) 309-332.

[6] Y. Z. Boutros, M. B. Abd-el-Malek, N. A. Badran, Group theoretic approach for solving time-independent freeconvective boundary layer flow on a nonisothermal vertical flat plate, Arch. Mech. 42 (1990) 377-395.

[7] M. Kumari, A. Slaouti, H. S. Takhar, S. Nakamura, G. Nath, Unsteady free convection flow over a continuous moving vertical surface, Acta Mech. 116 (1996) 75-82.

[8] Bataller, R. C., 2008, "Radiation Effects for the Blasius and Sakiadis Flows With a Convective Surface Boundary Condition," Appl. Math. Comput., 206, pp. 832-840.

[9] Hong. J. T., Tien, C. L. and Kaviany, M (1985), Non-Darcian effects on vertical-plate natural convection in porous media with high porosities, Int. J. Heat Mass Transfer Vol.28, pp. 2149-2175.

[10] Chen. C. K. and Lin, C. R. (1995), Natural convection from an isothermal vertical surface embedded in a thermally stratified high-porosity medium, Int. J. Engg. Sci., Vol. 33, pp. 131-138.

[11] Jaisawal, B. S. and Soundalgekar, V. M. (2001), Oscillating plate temperature effects on a flow past an infinite vertical porous plate with constant suction and embedded in a porous medium, Heat Mass Transfer,Vol.37, pp. 125-131.

[12] B. C. Sakiadis, Flow and heat transfer in the boundary layer on a continuous moving surface, AIChE J. 7 (1) (1961) 26-28.

[13] B. C. Sakiadis, Boundary layer behavior on continuous solid surface, II. The boundary layer on a continuous flat surface, AIChE J.7 (1) (1961) 221-225.

[14] Andersson HI. Slip flow past a stretching surface. Acta Mech 2002; 158: 121-5.

[15] Wang CY. Flow due to a stretching boundary with partial slip -an exact solution of the Navier-Stokes equations. Chem Eng Sci 2002; 57: 3745-7. 
[16] F. K. Tsou, E. M. Sparrow, R. J. Glodstein, Flow and heat transfer in the boundary layer on a continuous moving surface, Int. J. Heat Mass Transfer 10 (1967) 219-235.

[17] Ali M. Yeakub, Hossain, M. M. Touhid, Similarity solution for unsteady laminar natural convection boundary layer flow around a vertical heated curvilinear surface, Int.j. of Appl. Math and Stat.(2012)0973_1377.

[18] A. Aziz, A similarity solution for laminar thermal boundary layer over a flat plate with a convective surface boundary condition, Commun. Nonlinear Sci. Numer. Simul. 14 (2009) 1064-1068.

[19] E. Magyari, Comment on "A similarity solution for laminar thermal boundary layer over a flat plate with a convective surface boundary condition" by A. Aziz, Nonlinear Sci. Numer. Simul. 142009 1064-1068, Nonlinear Sci. Numer. Simul. (2010), doi:10.1016/j.cnsns.2010.03.020.

[20] A. Ishak, Similarity solutions for flow and heat transfer over a permeable surface with convective boundary condition, Applied Mathematics and Computation 217 (2010) 837-842.
[21] O. D. Makinde and P. O. Olanrewaju, Buoyancy effects on thermal boundary layer over a vertical plate with a convective surface boundary condition, ASME Journal of Fluid Engineering 132 (2010) 044502 1-4.

[22] A. Raptis, C. Perdikis, H. S. Takhar, Effects of thermal radiation on MHD flow, Appl. Math. Comput. 153 (2004) 645-649.

[23] T. Hayat, Z. Abbas, M. Sajid, S. Asghar, The influence of thermal radiation on MHD flowof a second grade fluid, Int. J. Heat Mass Transfer 50 (2007) 931-941.

[24] Olanrewaju, P. O., Gbadeyan, J. A.,Hayat T, Effects of internal heat generation, thermal radiation, and buoyancy force on boundary layer over a vertical plate with a convective boundary condition, Can. J. Chem. Eng. 90 (2012) 1289-1294.

[25] A. J. Chamkha, "Hydromagnetic natural convection from an isothermal inclined surface adjacent to a thermally stratified porous medium," International Journal of Engineering Science, vol. 37, no. 10-11, pp. 975-986, 1997. 